\title{
NON-SPECTRAL FRACTAL MEASURES WITH FOURIER FRAMES
}

\author{
CHUN-KIT LAI AND YANG WANG
}

\begin{abstract}
We generalize the compatible tower condition given by Strichartz to the almost-Parseval-frame tower and show that non-trivial examples of almostParseval-frame tower exist. By doing so, we demonstrate the first singular fractal measure which has only finitely many mutually orthogonal exponentials (and hence it does not admit any exponential orthonormal bases), but it still admits Fourier frames.
\end{abstract}

\section{Contents}

1. Introduction

2. Existence of Almost-Parseval-frame tower 5

3. Construction of Fourier frames 8

4. Non-spectral measures 14

5. Appendix: Hausdorff dimension 17

$\begin{array}{ll}\text { References } & 19\end{array}$

\section{INTRODUCTION}

Let $\mu$ be a compactly supported Borel probability measure on $\mathbb{R}^{d}$. We say that $\mu$ is a frame spectral measure if there exists a collection of exponential functions $\left\{e^{2 \pi i\langle\lambda, x\rangle}\right\}_{\lambda \in \Lambda}$ such that there exists $0<A \leq B<\infty$ with

$$
A\|f\|_{2}^{2} \leq \sum_{\lambda \in \Lambda}\left|\int f(x) e^{-2 \pi i\langle\lambda, x\rangle} d \mu(x)\right|^{2} \leq B\|f\|_{2}^{2}, \forall f \in L^{2}(\mu) .
$$

Whenever such $\Lambda$ exists, $\left\{e^{2 \pi i\langle\lambda, x\rangle}\right\}_{\lambda \in \Lambda}$ is called a Fourier frame for $L^{2}(\mu)$ and $\Lambda$ is a frame spectrum for $\mu$. When $\mu$ admits an exponential orthonormal basis, we say that $\mu$ is a spectral measure and the corresponding frequency set $\Lambda$ is called a spectrum for $\mu$.

Frames on a general Hilbert space was introduced by Duffin and Schaeffer [DS] and it is now a fundamental building block in applied harmonic analysis. People

2010 Mathematics Subject Classification. Primary 28A25, 42A85, 42B05.

Key words and phrases. Fourier frame, frame spectral measures, fractal measures, almost Parseval frames, spectra. 
regard frames as "overcomplete basis" and because of its redundancy, it makes the reconstruction more robust to errors in data and it is now widely used in signal transmission and reconstruction. Reader may refer to [Chr] for the background of general frame theory and $[\mathrm{CK}]$ for some recent active topics.

One of the major hard problems in frame theory perhaps is constructing Fourier frames or exponential orthonomal bases in different measure space $L^{2}(\mu)$, particularly when $\mu$ is a singular measure without any atoms or people termed it as a "fractal measure" as the support is a fractal set. These constructions allow Fourier analysis to work on fractal space. This problem dates back to the time of Fuglede [Fu] who initiated the study and proposed the well-known spectral set conjecture. Although the conjecture was proved to be false by Tao $[\mathrm{T}]$, the conjecture has been extended into different facet and related questions are still being studied [W, IKT1, IKT2, K, KN]. Another major advance in which fractals were involved was due to Jorgensen and Pedersen [JP1], who discovered that the standard one-third Cantor measure is not a spectral measure, while the standard one-fourth Cantor measure is. Following the discovery, more fractal measures were found to be spectral by many others [St1, ŁaW1, DJ1]. Many unexpected properties of the Fourier bases were discovered [St2, DHS, DaHL]. While Fourier analysis appears to work perfectly on fractal spectral measures, for the measures which are non-spectral, it is natural to ask the following question.

(Q): Can a non-spectral fractal measure still admit some Fourier frames?

This question was possibly first proposed by Strichartz [St1, p.212]. In particular, there has been discussions asking whether specifically the one-third Cantor measure can be frame spectral. Although we are unable to settle the case of the one-third Cantor measure, the main purpose of this paper is to answer positively (Q) with explicit examples. (see Theorem 1.4).

(Q) in its absolutely continuous counterpart is trivial since every bounded Borel set $\Omega$ with positive finite Lebesgue measure can be covered by a square. The orthonormal basis on the square naturally induces a tight frame on $\Omega$. If $\mu=g(x) d x$ is a general absolutely continuous measure, a complete characterization on the density for $\mu$ to be frame spectral was also given by the first named author [Lai]. Such question becomes much more difficult if $\Omega$ is unbounded but still of finite measure as there cannot be any ad hoc "square-covering" argument to construct the Fourier frames. Despite the difficulty, it was recently solved to be positive by Nitzan et al [NOU] who used the recent solution of the celebrated Kadison-Singer conjecture [MSS].

Fractal measures are mostly supported on Lebesgue measure zero set, the situation is similar to unbounded sets of finite measures. However, it is even more complicated because if any such frame spectrum exists, there cannot be any Beurling density 
[DHSW, DaHL]. This prevents any weak convergence argument of discrete sets from happening. Furthermore, some fractal measures are known not to admit any Fourier frames if the measures are non-uniform on the support [DL1]. Intensive researches on this question [DHSW, DHW1, DHW2, DL1, DL2, HLL] has been going on and one major advance was obtained recently in [DL2]. Dutkay and Lai introduced the almost-Parseval-frame condition for the self-similar measure and proved that if such condition is satisfied, the self-similar measure admits a Fourier frame. We slightly modify the definition as below to suit the need in the paper.

Definition 1.1. Let $\epsilon_{j}$ be such that $0 \leq \epsilon_{j}<1$ and $\sum_{j=1}^{\infty} \epsilon_{j}<\infty$. We say that $\left\{\left(N_{j}, B_{j}\right)\right\}$ is an almost-Parseval-frame tower associated to $\left\{\epsilon_{j}\right\}$ if

(1) $N_{j}$ are integers and $N_{j} \geq 2$ for all $j$;

(2) $B_{j} \subset\left\{0,1, \ldots, N_{j}-1\right\}$ and $0 \in B_{j}$ for all $j$;

(3) Let $M_{j}:=\# B_{j}$. There exists $L_{j} \subset \mathbb{Z}$ (with $0 \in L_{j}$ ) such that for all $j$,

$$
\left(1-\epsilon_{j}\right)^{2} \sum_{b \in B_{j}}\left|w_{b}\right|^{2} \leq \sum_{\lambda \in L_{j}}\left|\frac{1}{\sqrt{M_{j}}} \sum_{b \in B_{j}} w_{b} e^{-2 \pi i b \lambda / N_{j}}\right|^{2} \leq\left(1+\epsilon_{j}\right)^{2} \sum_{b \in B_{j}}\left|w_{b}\right|^{2}
$$

for all $\mathbf{w}=\left(w_{b}\right)_{b \in B_{j}} \in \mathbb{C}^{M_{j}}$. Letting the matrix $\mathcal{F}_{j}=\frac{1}{\sqrt{M_{j}}}\left[e^{2 \pi i b \lambda / N_{j}}\right]_{\lambda \in L_{j}, b \in B_{j}}$ and $\|\cdot\|$ the standard Euclidean norm, (1.1) is equivalent to

$$
\left(1-\epsilon_{j}\right)\|\mathbf{w}\| \leq\left\|\mathcal{F}_{j} \mathbf{w}\right\| \leq\left(1+\epsilon_{j}\right)\|\mathbf{w}\|
$$

for all $\mathbf{w} \in \mathbb{C}^{M_{j}}$.

Whenever $\left\{L_{j}\right\}_{j \in \mathbb{Z}}$ exists, we call $\left\{L_{j}\right\}_{j \in \mathbb{Z}}$ a pre-spectrum for the almost-Parsevalframe tower. We define the following measures associated to an almost-Parsevalframe tower.

$$
\nu_{j}=\frac{1}{M_{j}} \sum_{b \in B_{j}} \delta_{b / N_{1} N_{2} \ldots N_{j}}
$$

(we denote by $\delta_{a}$ the Dirac measure supported on $a$ ) and

$$
\mu=\nu_{1} * \nu_{2} * \ldots
$$

Roughly speaking, almost-Parseval-frame towers ensure every finite level approximated measure of the fractal is a frame spectral measure. Moreover, the frame bounds remain finite under iterations. Once all finite level has a frame with uniform frame bound, we take the weak limit under a mild condition so that fractal singular measure is also frame-spectral.

When all $\epsilon_{j}=0$, the condition is equivalent to the compatible tower condition introduced by Strichartz [St1]. This is known to be the key condition to construct fractal spectral measures. The measures in (1.3) are also known as Moran-type measures. These measures have been widely used in multifractal analysis [FL, FLW], 
harmonic analysis, particularly the construction of Salem sets [ŁP1, ŁP2]. Some Moran-type measures were found to be spectral $[\mathrm{AH}]$ and it was found later spectral Moran measures have a far reaching consequence in understanding the spectral set conjecture [GL] and Hausdorff dimension of the support of the spectral measures [DaS]. We note that Moran-type measures covers self-similar measures because if there exists an integer $N \geq 2$ and a set $B \subset\{0,1, \ldots, N-1\}$ such that

$$
N_{j}=N^{n_{j}}, B_{j}=B+N B+\ldots+N^{n_{j}-1} B
$$

then the associated measure is the self-similar measure. In particular if $N=3$ and $B=\{0,2\}, \mu$ is the standard one-third Cantor measure. In such situation, the almost-Parseval-frame tower is called self-similar.

In [DL2], it was proved if the almost-Parseval-frame tower is self-similar, then the self-similar measure induced will admit an Fourier frame. However, there was no example of such towers for which $\epsilon_{j}>0$. In this paper, we relax the self-similar restriction and produce the first example almost-Parseval-frame tower whose $\epsilon_{j}>0$. We prove

Theorem 1.2. Let $N_{j}$ and $M_{j}$ be positive integers satisfying

$$
N_{j}=M_{j} K_{j}+\alpha_{j}
$$

for some integer $K_{j}$ and $0 \leq \alpha_{j}<M_{j}$ with

$$
\sum_{j=1}^{\infty} \frac{\alpha_{j} \sqrt{M_{j}}}{K_{j}}<\infty .
$$

Define

$$
B_{j}=\left\{0, K_{j}, \ldots,\left(M_{j}-1\right) K_{j}\right\}, L_{j}=\left\{0,1, \ldots, M_{j}-1\right\} .
$$

Then $\left(N_{j}, B_{j}\right)$ forms an almost-Parseval-frame tower associated with

$$
\epsilon_{j}=\frac{2 \pi \alpha_{j} \sqrt{M_{j}}}{K_{j}}
$$

and its pre-spectrum is $\left\{L_{j}\right\}$.

We then extend the result of [DL2] to general almost-Parseval-frame tower. For the measure $\mu$ defined in (1.3), we let

$$
\mu_{n}=\nu_{1} * \ldots * \nu_{n}, \mu_{>n}=\nu_{n+1} * \nu_{n+2} * \ldots
$$

so that $\mu=\mu_{n} * \mu_{>n}$. Define also the Fourier transform of a measure $\mu$ in an usual way.

$$
\widehat{\mu}(\xi)=\int e^{-2 \pi i \xi x} d \mu(x)
$$


Theorem 1.3. (a) Suppose that $\left\{\left(N_{j}, B_{j}\right)\right\}$ is an almost-Parseval-frame tower associated with $\left\{\epsilon_{j}\right\}$ and $\left\{L_{j}\right\}_{j=1}^{\infty}$. Let

$$
\mathbf{L}_{n}=L_{1}+N_{1} L_{2}+\ldots+\left(N_{1} \ldots N_{n-1}\right) L_{n}, \text { and } \Lambda=\bigcup_{n=1}^{\infty} \mathbf{L}_{n}
$$

If

$$
\delta(\Lambda):=\inf _{n} \inf _{\lambda \in \mathbf{L}_{n}}\left|\widehat{\mu_{>n}}(\lambda)\right|^{2}>0,
$$

then the measure $\mu$ in (1.3) admits a Fourier frame with frame spectrum $\Lambda$.

(b) For the almost-Parseval-frame tower constructed in Theorem 1.2, the associated $\Lambda$ satisfies $\delta(\Lambda)>0$ and hence the measure $\mu$ is a frame spectral measure.

In the end, using the theorems above, we construct the first kind of the following examples:

Theorem 1.4. There exists non-spectral fractal measure with only finitely many orthogonal exponentials, but it still admits Fourier frames.

We organize our paper as follows: In section 2, we prove the existence of the almost-Parseval-frame tower and prove Theorem 1.2. In Section 3, we construct the Fourier frame given the tower and prove Theorem 1.3. In Section 4, we construct the non-spectral measures with Fourier frames. In the appendix, we study the Hausdorff dimension of the support.

\section{Existence of Almost-Parseval-Frame tower}

Let $A$ be an $n \times n$ matrix. We define the operator norm of $A$ to be

$$
\|A\|=\max _{\|\mathbf{x}\|=1}\|A \mathbf{x}\|
$$

and the Frobenius norm of $A$ to be

$$
\|A\|_{F}=\sqrt{\sum_{i=1}^{n} \sum_{j=1}^{n}\left|a_{i, j}\right|^{2}}
$$

It follows easily from Cauchy-Schwarz inequality that $\|A\|_{2} \leq\|A\|_{F}$.

For $N_{j}$ and $M_{j}$ satisfying (1.4) and (1.5) and for $B_{j}$ and $L_{j}$ defined in (1.6), We let

$$
\mathcal{F}_{j}=\frac{1}{\sqrt{M_{j}}}\left[e^{2 \pi i b \lambda / N_{j}}\right]_{\lambda \in L_{j}, b \in B_{j}}, \mathcal{H}_{j}=\frac{1}{\sqrt{M_{j}}}\left[e^{2 \pi i b \lambda / M_{j} K_{j}}\right]_{\lambda \in L_{j}, b \in B_{j}} .
$$

Lemma 2.1. $\mathcal{H}_{n}$ is a unitary matrix. i.e. $\left\|\mathcal{H}_{n} \mathbf{x}\right\|=\|\mathbf{x}\|$. 
Proof. Let $b=m \in L_{j}$ and $\lambda=n K_{j} \in B_{j}$, for $m, n=0,1, \ldots, M_{j}-1$. It follows directly that $e^{2 \pi i b \lambda / M K_{n}}=e^{2 \pi i m n / M_{j}}$. Hence,

$$
\mathcal{H}_{j}=\frac{1}{\sqrt{M_{j}}}\left[e^{2 \pi i m n / M_{j}}\right]_{m, n=0, \ldots, M_{j}-1}
$$

which is the standard Fourier matrix of order $M_{j}$. Thus, $\mathcal{H}_{j}$ is unitary.

Proof of Theorem 1.2. We first show that for any $j>0$,

$$
\left\|\mathcal{F}_{j}-\mathcal{H}_{j}\right\| \leq \frac{2 \pi \alpha_{j} \sqrt{M_{j}}}{K_{j}}
$$

To see this, We note that

$$
\left\|\mathcal{F}_{j}-\mathcal{H}_{j}\right\|^{2} \leq\left\|\mathcal{F}_{j}-\mathcal{H}_{j}\right\|_{F}^{2}=\frac{1}{M_{j}} \sum_{b \in B_{j}} \sum_{\lambda \in L_{j}}\left|e^{2 \pi i b \lambda / N_{j}}-e^{2 \pi i b \lambda / M_{j} K_{j}}\right|^{2} .
$$

We now estimate the difference of the exponentials inside the summation. Recall that for any $\theta_{1}, \theta_{2}$,

$$
\left|e^{i \theta_{1}}-e^{i \theta_{2}}\right|=\left|e^{i\left(\theta_{1}-\theta_{2}\right)}-1\right| \leq\left|\theta_{1}-\theta_{2}\right|
$$

This implies that

$$
\begin{aligned}
\left|e^{2 \pi i b \lambda / N_{j}}-e^{2 \pi i b \lambda / M_{j} K_{j}}\right|^{2} & \leq\left|\frac{2 \pi b \lambda}{N_{j}}-\frac{2 \pi b \lambda}{M_{j} K_{j}}\right|^{2} \\
& =4 \pi^{2} \frac{b^{2} \lambda^{2} \alpha_{j}^{2}}{M_{j}^{2} K_{j}^{2} N_{j}^{2}} \quad\left(\text { by } N_{j}=M_{j} K_{j}+\alpha_{j}\right) \\
& \leq 4 \pi^{2} \frac{M_{j}^{2} \alpha_{j}^{2}}{N_{j}^{2}} \quad\left(\text { by } b \leq M_{j} K_{j} \text { and } \lambda \leq M_{j}\right)
\end{aligned}
$$

Hence, from (2.2),

$$
\begin{aligned}
\left\|\mathcal{F}_{j}-\mathcal{H}_{j}\right\|^{2} & \leq \frac{1}{M_{j}} \sum_{b \in B_{j}} \sum_{\lambda \in L_{j}} 4 \pi^{2} \frac{M_{j}^{2} \alpha_{j}^{2}}{N_{j}^{2}} \\
& =4 \pi^{2} \frac{M_{j}^{3} \alpha_{j}^{2}}{N_{j}^{2}} \\
& =4 \pi^{2} \frac{M_{j} \alpha_{j}^{2}}{\left(K_{j}+\alpha_{j} / M_{j}\right)^{2}}
\end{aligned}
$$

As $\alpha_{j} \geq 0,\left\|\mathcal{F}_{j}-\mathcal{H}_{j}\right\|^{2} \leq 4 \pi^{2} \alpha_{j}^{2} M_{j} / K_{j}^{2}$ and thus (2.1) follows by taking square root.

We now show that $\left\{\left(N_{j}, B_{j}\right)\right\}$ forms an almost-Parseval-frame tower with prespectrum $L_{j}$. The first two conditions for the almost-Parseval-frame tower are clearly 
satisfied. To see the last condition, we recall that $\epsilon_{j}=2 \pi \sqrt{M_{j}} \alpha_{j} / K_{j}$. From the triangle inequality and (2.1), we have

$$
\begin{aligned}
\left\|\mathcal{F}_{j} \mathbf{w}\right\| & \leq\left\|\mathcal{H}_{j} \mathbf{w}\right\|+\left\|\mathcal{F}_{j}-\mathcal{H}_{j}\right\|\|\mathbf{w}\| \\
& \leq\left(1+\frac{2 \pi \alpha_{j} \sqrt{M_{j}}}{K_{j}}\right)\|\mathbf{w}\|=\left(1+\epsilon_{j}\right)\|\mathbf{w}\| .
\end{aligned}
$$

Similarly, for the lower bound,

$$
\begin{aligned}
\left\|\mathcal{F}_{j} \mathbf{w}\right\| & \geq\left\|\mathcal{H}_{j} \mathbf{w}\right\|-\left\|\mathcal{F}_{j}-\mathcal{H}_{j}\right\|\|\mathbf{w}\| \\
& \geq\left(1-\frac{2 \pi \alpha_{j} \sqrt{M_{j}}}{K_{j}}\right)\|\mathbf{w}\|=\left(1-\epsilon_{j}\right)\|\mathbf{w}\| .
\end{aligned}
$$

Thus, from (1.2), the last condition follows and $\left(N_{j}, B_{j}\right)$ satisfies the almost-Parsevalframe condition associated with $\left\{\epsilon_{j}\right\}$ and $\sum_{j=1}^{\infty} \epsilon_{j}<\infty$ is guaranteed by (1.5) in the assumption.

Remark 2.2. In view of (2.3), condition (1.5) can be replaced by a weaker condition

$$
\sum_{j=1}^{\infty} \frac{\alpha_{j} \sqrt{M_{j}}}{K_{j}+\alpha_{j} / M_{j}}<\infty
$$

(1.5) would be enough for the convenience of our discussion. It is also worth to note that if all $\alpha_{j}=0$, then the the matrices $\mathcal{F}_{j}=\mathcal{H}_{j}$ are reduced to the Hadamard matrices. The associated measures are all spectral measures. see e.g. [AH].

We end this section by illustrating some explicit examples of Theorem 1.2.

Example 2.3. Let $p$ be an odd prime and suppose that $N_{j}=p^{j}$. Let $M_{j}=2$ for all $j$. Then it is clear that $N_{j}=2 K_{j}+1$ for some $K_{j}$. In this case,

$$
\sum_{j=1}^{\infty} \frac{\alpha_{j} \sqrt{M_{j}}}{K_{j}}=\sum_{j=1}^{\infty} \frac{\sqrt{2}}{K_{j}}=\sum_{j=1}^{\infty} \frac{2 \sqrt{2}}{p^{j}-1}<\infty .
$$

Thus $N_{j}=p^{j}$ and $B_{j}=\left\{0, K_{j}\right\}$ forms an almost-Parseval-frame tower with prespectrum $L_{j}=\{0,1\}$ for all $j$.

Example 2.4. For $0 \leq \beta<2, \gamma \geq 0$ and $N \geq 1$ such that

$$
N(1-\beta / 2-\gamma)>1 \text {, }
$$

let $K_{j}, M_{j}, \alpha_{j}$ be integers $K_{j} \geq j^{N}, M_{j} \leq K_{j}^{\beta}$ and $\alpha_{j} \leq K_{j}^{\gamma}$. Then

$$
\sum_{j=1}^{\infty} \frac{\alpha_{j} \sqrt{M_{j}}}{K_{j}} \leq \sum_{j=1}^{\infty} \frac{K_{j}^{\gamma} K_{j}^{\beta / 2}}{K_{j}}=\sum_{j=1}^{\infty} \frac{1}{K_{j}^{1-\gamma-\beta / 2}} \leq \sum_{j=1}^{\infty} \frac{1}{j^{N(1-\gamma-\beta / 2)}}<\infty .
$$

Hence, $N_{j}=K_{j} M_{j}+\alpha_{j}$ and $B_{j}=\left\{0, K_{j}, \ldots,\left(M_{j}-1\right) K_{j}\right\}$ satisfies the almostParseval-frame condition. 


\section{Construction of Fourier frames}

In this section, we consider the almost-Parseval-frame tower defined in Section 1 and show that the measure $\mu$ defined in (1.3) is a frame spectral measure. We first recall some notations.

$$
\nu_{j}=\frac{1}{M_{j}} \sum_{b \in B_{j}} \delta_{b / N_{1} \ldots N_{j}}, \text { and } \mu=\nu_{1} * \nu_{2} \ldots
$$

We define

$$
\mu_{n}=\nu_{1} * \ldots * \nu_{n}, \mu_{>n}=\nu_{n+1} * \nu_{n+2} * \ldots
$$

so that $\mu=\mu_{n} * \mu_{>n}$. It is also direct to see that the support of $\mu$ is the compact set

$$
K_{\mu}=\left\{\sum_{j=1}^{\infty} \frac{b_{j}}{N_{1} \ldots N_{j}}: b_{j} \in B_{j} \text { for all } j\right\} .
$$

We also consider the first $n^{\text {th }}$-partial sum in $K_{\mu}$ and denote it by

$$
\mathbf{B}_{n}=\frac{1}{N_{1}} B_{1}+\frac{1}{N_{1} N_{2}} B_{2}+\ldots+\frac{1}{N_{1} N_{2} \ldots N_{n}} B_{n}
$$

which is the support of $\mu_{n}$. For the $\left\{L_{j}\right\}_{j \in \mathbb{Z}}$ in the tower, we consider

$$
\mathbf{L}_{n}=L_{1}+N_{1} L_{2}+\ldots+\left(N_{1} \ldots N_{n-1}\right) L_{n} .
$$

Proposition 3.1. For any $n \geq 1$, let $\mathbf{M}_{n}=\prod_{j=1}^{n} M_{j}$ we have

$$
\left(\prod_{j=1}^{n}\left(1-\epsilon_{j}\right)\right)^{2}\|\mathbf{w}\|^{2} \leq \sum_{\lambda \in \mathbf{L}_{n}}\left|\frac{1}{\sqrt{\mathbf{M}_{n}}} \sum_{\mathbf{b} \in \mathbf{B}_{n}} w_{\mathbf{b}} e^{-2 \pi i \mathbf{b} \lambda}\right|^{2} \leq\left(\prod_{j=1}^{n}\left(1+\epsilon_{j}\right)\right)^{2}\|\mathbf{w}\|^{2}
$$

for any $\mathbf{w}=\left(w_{\mathbf{b}}\right)_{\mathbf{b} \in \mathbf{B}_{n}} \in \mathbb{C}^{M_{1} \ldots M_{n}}$

Proof. We prove it by mathematical induction. When $n=1$, it is the almostParseval condition for $\left(N_{1}, B_{1}\right)$ so the statement is true trivially. Assume now the inequality is true for $n-1$. Then we decompose $\mathbf{b} \in \mathbf{B}_{n}$ and $\lambda \in \mathbf{L}_{n}$ by

$$
\mathbf{b}=\frac{1}{N_{1} \ldots N_{n}} b_{n}+\mathbf{b}_{n-1}, \quad \lambda=\lambda_{n-1}+N_{1} \ldots N_{n-1} l_{n},
$$

where $b_{n} \in B_{n}, \mathbf{b}_{n-1} \in \mathbf{B}_{n-1}, \lambda_{n-1} \in \mathbf{L}_{n-1}$ and $l_{n} \in L_{n}$. Now, we have

$$
\begin{aligned}
& \sum_{\lambda \in \mathbf{L}_{n}}\left|\sum_{\mathbf{b} \in \mathbf{B}_{n}} w_{\mathbf{b}} e^{-2 \pi i \mathbf{b} \lambda}\right|^{2} \\
= & \sum_{\lambda_{n-1} \in \mathbf{L}_{n-1}} \sum_{l_{n} \in L_{n}}\left|\sum_{\mathbf{b}_{n-1} \in \mathbf{B}_{n-1}} \sum_{b_{n} \in B_{n}} \frac{1}{\sqrt{\mathbf{M}_{n}}} w_{\mathbf{b} b_{n}} e^{-2 \pi i\left(\frac{1}{N_{1} \ldots N_{n}} b_{n}+\mathbf{b}_{n-1}\right) \cdot\left(\lambda_{n-1}+N_{1} \ldots N_{n-1} l_{n}\right)}\right|^{2} .
\end{aligned}
$$


Note that $\mathbf{b}_{n-1} \cdot\left(N_{1} \ldots N_{n-1}\right) l_{n}$ is always an integer, the right hand side above can be written as

$$
\sum_{\lambda_{n-1} \in \mathbf{L}_{n-1}} \sum_{l_{n} \in L_{n}}\left|\sum_{b_{n} \in B_{n}} \frac{1}{\sqrt{M_{n}}}\left(\sum_{\mathbf{b}_{n-1} \in \mathbf{B}_{n-1}} \frac{1}{\sqrt{\mathbf{M}_{n-1}}} w_{\mathbf{b}_{n-1} b_{n}} e^{-2 \pi i\left(\frac{1}{N_{1} \ldots N_{n}} b_{n}+\mathbf{b}_{n-1}\right) \cdot \lambda_{n-1}}\right) e^{-2 \pi i b_{n} l_{n} / N_{n}}\right|^{2}
$$

Using the almost-Parseval-frame condition for $\left(N_{n}, B_{n}\right)$ and also the induction hypothesis, this term

$$
\begin{aligned}
& \leq\left(1+\epsilon_{n}\right)^{2} \sum_{\lambda_{n-1} \in \mathbf{L}_{n-1}} \sum_{b_{n} \in B_{n}}\left|\sum_{\mathbf{b}_{n-1} \in \mathbf{B}_{n-1}} \frac{1}{\sqrt{\mathbf{M}_{n-1}}} w_{\mathbf{b}_{n-1} b_{n}} e^{-2 \pi i\left(\frac{1}{N_{1} \ldots N_{n}} b_{n}+\mathbf{b}_{n-1}\right) \cdot \lambda_{n-1}}\right|^{2} \\
& =\left(1+\epsilon_{n}\right)^{2} \sum_{b_{n} \in B_{n}} \sum_{\lambda_{n-1} \in \mathbf{L}_{n-1}}\left|\sum_{\mathbf{b}_{n-1} \in \mathbf{B}_{n-1}} \frac{1}{\sqrt{\mathbf{M}_{n-1}}} w_{\mathbf{b}_{n-1} b_{n}} e^{-2 \pi i \mathbf{b}_{n-1} \cdot \lambda_{n-1}}\right|^{2} \\
& \leq\left(\prod_{j=1}^{n}\left(1+\epsilon_{j}\right)\right)^{2} \sum_{b_{n} \in B_{n}} \sum_{\mathbf{b}_{n-1} \in \mathbf{B}_{n-1}}\left|w_{\mathbf{b}_{n-1} b_{n}}\right|^{2} \\
& =\left(\prod_{j=1}^{n}\left(1+\epsilon_{j}\right)\right)^{2}\|\mathbf{w}\|^{2} .
\end{aligned}
$$

This completes the proof of the upper bound and the proof of the lower bound is analogous.

We now decompose $K_{\mu}$ as

$$
K_{\mu}=\bigcup_{\mathbf{b} \in \mathbf{B}_{n}}\left(\mathbf{b}+K_{\mu, n}\right)
$$

where

$$
K_{\mu, n}=\left\{\sum_{j=n+1}^{\infty} \frac{b_{j}}{N_{1} \ldots N_{j}}: b_{j} \in B_{j} \text { for all } j\right\} .
$$

Denote by $K_{\mathbf{b}}=\mathbf{b}+K_{\mu, n}$ and $\mathbf{1}_{K_{\mathbf{b}}}$ the characteristic function of $K_{\mathbf{b}}$. Let

$$
\mathcal{S}_{n}=\left\{\sum_{\mathbf{b} \in \mathbf{B}_{n}} w_{\mathbf{b}} \mathbf{1}_{K_{\mathbf{b}}}: w_{\mathbf{b}} \in \mathbb{C}\right\}
$$

$\mathcal{S}_{n}$ denotes the collection of all $n^{\text {th }}$ level step functions on $K_{\mu}$. As

$$
K_{\mu, n}=\bigcup_{b \in B_{n+1}}\left(\frac{b}{N_{1} \ldots N_{n+1}}+K_{\mu, n+1}\right),
$$

we have $\mathcal{S}_{1} \subset \mathcal{S}_{2} \subset \ldots$. Let also

$$
\mathcal{S}=\bigcup_{\substack{n=1 \\ 9}}^{\infty} \mathcal{S}_{n}
$$


It is clear that $\mathcal{S}$ forms a dense set of functions in $L^{2}(\mu)$.

Lemma 3.2. Let $f=\sum_{\mathbf{b} \in \mathbf{B}_{n}} w_{\mathbf{b}} \mathbf{1}_{K_{\mathbf{b}}} \in \mathcal{S}_{n}$. Then

$$
\begin{aligned}
\int|f|^{2} d \mu & =\frac{1}{\mathbf{M}_{n}} \sum_{\mathbf{b} \in \mathbf{B}_{n}}\left|w_{\mathbf{b}}\right|^{2} . \\
\int f(x) e^{-2 \pi i \lambda x} d \mu(x) & =\frac{1}{\mathbf{M}_{n}} \widehat{\mu_{>n}}(\lambda) \sum_{\mathbf{b} \in \mathbf{B}_{n}} w_{\mathbf{b}} e^{-2 \pi i \mathbf{b} \lambda} .
\end{aligned}
$$

Here $\mathbf{M}_{n}=M_{1} \ldots M_{n}$.

Proof. As $K_{\mathbf{b}}$ and $K_{\mathbf{b}}^{\prime}$ has either empty intersection or intersects at most one point, taking $\mu$-measure on (3.1), we obtain $\mu\left(K_{\mathbf{b}}\right)=1 / \mathbf{M}_{n}$. (3.2) follows from a direct computation. For (3.3), we use $\mu=\mu_{n} * \mu_{>n}$ and we have

$$
\begin{aligned}
\int f(x) e^{-2 \pi i \lambda x} d \mu(x) & =\sum_{\mathbf{b} \in \mathbf{B}_{n}} w_{\mathbf{b}} \int \mathbf{1}_{K_{\mathbf{b}}}(x) e^{-2 \pi i \lambda x} d\left(\mu_{n} * \mu_{>n}(x)\right) \\
& =\sum_{\mathbf{b} \in \mathbf{B}_{n}} w_{\mathbf{b}} \int \mathbf{1}_{\mathbf{b}+K_{\mu, n}}(x+y) e^{-2 \pi i \lambda(x+y)} d \mu_{n}(x) d \mu_{>n}(y) .
\end{aligned}
$$

Note that $\mu_{>n}$ is supported on $K_{\mu, n}$ and $K_{\mathbf{b}}$ and $K_{\mathbf{b}}^{\prime}$ has either empty intersection or intersects at most one point. The above is equal to

$$
\begin{aligned}
& =\sum_{\mathbf{b} \in \mathbf{B}_{n}} w_{\mathbf{b}} \frac{1}{\mathbf{M}_{n}} \int \mathbf{1}_{\mathbf{b}+K_{\mu, n}}(\mathbf{b}+y) e^{-2 \pi i \lambda(\mathbf{b}+y)} d \mu_{>n}(y) \\
& =\sum_{\mathbf{b} \in \mathbf{B}_{n}} w_{\mathbf{b}} \frac{1}{\mathbf{M}_{n}} e^{-2 \pi i \lambda \mathbf{b}} \int e^{-2 \pi i \lambda y} d \mu_{>n}(y) \\
& =\frac{1}{\mathbf{M}_{n}} \widehat{\mu_{>n}}(\lambda) \sum_{\mathbf{b} \in \mathbf{B}_{n}} w_{\mathbf{b}} e^{-2 \pi i \mathbf{b} \lambda} .
\end{aligned}
$$

The lemma follows.

Let

$$
\Lambda=\bigcup_{n=1}^{\infty} \mathbf{L}_{n} .
$$

As $0 \in L$, the sets in the union is an increasing union. We now define the following quantity

$$
\delta_{n_{0}}(\Lambda)=\inf _{n \geq n_{0}} \inf _{\lambda \in \mathbf{L}_{n}}\left|\widehat{\mu_{>n}}(\lambda)\right|^{2}
$$

for some $n_{0} \geq 1$. The following theorem gives a sufficient condition for $\Lambda$ to be a Fourier frame for $\mu$. 
Theorem 3.3. Suppose that $\left(N_{j}, B_{j}\right)$ is an almost-Parseval-frame tower and $\mu$ be the associated measure. Let $L$ be the associated spectrum for the tower and $\Lambda$ defined (3.4) satisfies $\delta_{n_{0}}(\Lambda)>0$. Then $\mu$ admits a Fourier frame $E(\Lambda)$ with lower and upper frame bounds respectively equal

$$
\delta(\Lambda)\left(\prod_{j=1}^{\infty}\left(1-\epsilon_{j}\right)\right)^{2},\left(\prod_{j=1}^{\infty}\left(1+\epsilon_{j}\right)\right)^{2} .
$$

Proof. To check the Fourier frame inequality holds, it suffices to show that it is true for a dense set of functions in $L^{2}(\mu)$ [Chr, Lemma 5.1.7], in which we will check it for step functions in $\mathcal{S}$. Moreover, since $\mathcal{S}_{n}$ is an increasing union of sets, we consider $f=\sum_{\mathbf{b} \in \mathbf{B}_{n}} w_{\mathbf{b}} \mathbf{1}_{K_{\mathbf{b}}} \in \mathcal{S}_{n}$ with $n \geq n_{0}$. By Lemma 3.2, we have

$$
\begin{aligned}
\sum_{\lambda \in \mathbf{L}_{n}}\left|\int f(x) e^{-2 \pi i \lambda x} d \mu(x)\right|^{2} & =\sum_{\lambda \in \mathbf{L}_{n}}\left|\frac{1}{\mathbf{M}_{n}} \widehat{\mu_{>n}}(\lambda) \sum_{\mathbf{b} \in \mathbf{B}_{n}} w_{\mathbf{b}} e^{-2 \pi i \mathbf{b} \lambda}\right|^{2} \\
& =\frac{1}{\mathbf{M}_{n}} \sum_{\lambda \in \mathbf{L}_{n}}\left|\widehat{\mu_{>n}}(\lambda)\right|^{2}\left|\frac{1}{\sqrt{\mathbf{M}_{n}}} \sum_{\mathbf{b} \in \mathbf{B}_{n}} w_{\mathbf{b}} e^{-2 \pi i \mathbf{b} \lambda}\right|^{2}
\end{aligned}
$$

Note that $\delta(\Lambda) \leq\left|\widehat{\mu_{>n}}(\lambda)\right|^{2} \leq 1$. By Proposition 3.1, we have this implies that

$$
\frac{1}{\mathbf{M}_{n}} \delta(\Lambda)\left(\prod_{j=1}^{n}\left(1-\epsilon_{j}\right)\right)^{2}\|\mathbf{w}\|^{2} \leq \sum_{\lambda \in \mathbf{L}_{n}}\left|\int f(x) e^{-2 \pi i \lambda x} d \mu(x)\right|^{2} \leq \frac{1}{\mathbf{M}_{n}}\left(\prod_{j=1}^{n}\left(1+\epsilon_{j}\right)\right)^{2}\|\mathbf{w}\|^{2} .
$$

Using Lemma 3.2 again, we have

$\delta(\Lambda)\left(\prod_{j=1}^{n}\left(1-\epsilon_{j}\right)\right)^{2} \int|f|^{2} d \mu \leq \sum_{\lambda \in \mathbf{L}_{n}}\left|\int f(x) e^{-2 \pi i \lambda x} d \mu(x)\right|^{2} \leq\left(\prod_{j=1}^{n}\left(1+\epsilon_{j}\right)\right)^{2} \int|f|^{2} d \mu$.

To complete the proof, we note that for all $m>n, f \in \mathcal{S}_{n} \subset \mathcal{S}_{m}$, the inequality can also be written as

$\delta(\Lambda)\left(\prod_{j=1}^{m}\left(1-\epsilon_{j}\right)\right)^{2} \int|f|^{2} d \mu \leq \sum_{\lambda \in \mathbf{L}_{m}}\left|\int f(x) e^{-2 \pi i \lambda x} d \mu(x)\right|^{2} \leq\left(\prod_{j=1}^{m}\left(1+\epsilon_{j}\right)\right)^{2} \int|f|^{2} d \mu$,

for all $f \in \mathcal{S}_{n}$. Taking $m$ to infinity, we have

$\delta(\Lambda)\left(\prod_{j=1}^{\infty}\left(1-\epsilon_{j}\right)\right)^{2} \int|f|^{2} d \mu \leq \sum_{\lambda \in \Lambda}\left|\int f(x) e^{-2 \pi i \lambda x} d \mu(x)\right|^{2} \leq\left(\prod_{j=1}^{\infty}\left(1+\epsilon_{j}\right)\right)^{2} \int|f|^{2} d \mu$.

Note that the frame bounds are finite since $\sum_{j=1}^{\infty} \epsilon_{j}<\infty$. This shows the frame inequality for any $f \in \mathcal{S}$. Hence, $E(\Lambda)$ is a Fourier frame for $L^{2}(\mu)$. 
We now show that the almost-Parseval-frame tower constructed in Theorem 1.2 satisfies $\delta(\Lambda)>0$. Recall that

$$
N_{j}=M_{j} K_{j}+\alpha_{j}
$$

with $B_{j}=\left\{0, K_{j}, \ldots,\left(M_{j}-1\right) K_{j}\right\}$ and $L_{j}=\left\{0,1, \ldots, M_{j}-1\right\}$. The associated measure is given by

$$
\mu=\nu_{1} * \nu_{2} * \ldots, \text { and } \nu_{j}=\frac{1}{M_{j}} \sum_{b \in B_{j}} \delta_{j / N_{1} \ldots N_{j}} .
$$

The Fourier transform is given by

$$
\widehat{\mu}(\xi)=\prod_{j=1}^{\infty} \widehat{\nu_{j}}(\xi)=\prod_{j=1}^{\infty}\left[\frac{1}{M_{j}} \sum_{k=0}^{M_{j}-1} e^{-2 \pi i k K_{j} \xi / N_{1} \ldots N_{j}}\right]
$$

It follows directly from summation of geometric series that

$$
\widehat{\nu_{j}}(\xi)= \begin{cases}\frac{1}{M_{j}} e^{\pi i c_{j}\left(M_{j}-1\right) \xi \frac{\sin \pi c_{j} M_{j} \xi}{\sin \pi c_{j} \xi},}, & \text { if } \xi \notin \frac{1}{c_{j}} \mathbb{Z} \\ 1, & \text { if } \xi \in \frac{1}{c_{j}} \mathbb{Z}\end{cases}
$$

where $c_{j}=K_{j} / N_{1} \ldots N_{j}$. We prove that

Proposition 3.4. With all the notation above, there exists $k_{0}$ such that $\Lambda=\bigcup_{k=1}^{\infty} \mathbf{L}_{k}$ satisfies

$$
\delta_{k_{0}}(\Lambda)=\inf _{k \geq k_{0}} \inf _{\lambda \in \mathbf{L}_{k}}\left|\widehat{\mu_{>k}}(\lambda)\right|^{2}>0
$$

where $\mathbf{L}_{k}=L_{1}+N_{1} L_{2}+\ldots+N_{1} \ldots N_{k-1} L_{k}$.

Proof. We note that

$$
\left|\widehat{\mu_{>k}}(\lambda)\right|^{2}=\prod_{j=1}^{\infty}\left|\widehat{\nu_{k+j}}(\lambda)\right|^{2}=\prod_{j=1}^{\infty}\left|\frac{1}{M_{k+j}} \sum_{\ell=0}^{M_{k+j}-1} e^{-2 \pi i \ell K_{k+j} \lambda /\left(N_{1} \ldots N_{k} N_{k+1} \ldots N_{k+j}\right)}\right|^{2}
$$

For any $\lambda \in \mathbf{L}_{k}$ for which the terms $\left|\widehat{\nu_{k+j}}(\lambda)\right|^{2}<1$, we have

$$
\left|\frac{1}{M_{k+j}} \sum_{k=0}^{M_{j}-1} e^{-2 \pi i k K_{j} \lambda /\left(N_{1} \ldots N_{k} N_{k+1} \ldots N_{k+j}\right)}\right|^{2}=\left|\frac{1}{M_{k+j}} \frac{\sin \pi c_{k+j} M_{k+j} \lambda}{\sin \pi c_{k+j} \lambda}\right|^{2} .
$$

Using the elementary estimate $\sin x \leq x$ and $\sin x \geq x-x^{3} / 3$ !, we have

$$
\left|\frac{1}{M_{k+j}} \frac{\sin \pi c_{k+j} M_{k+j} \lambda}{\sin \pi c_{k+j} \lambda}\right|^{2} \geq\left|\frac{\sin \left(\pi c_{k+j} M_{k+j} \lambda\right)}{\pi c_{k+j} M_{k+j} \lambda}\right|^{2}=\left(1-\frac{\left(\pi c_{k+j} M_{k+j} \lambda\right)^{2}}{3 !}\right)^{2}
$$

Recall that $c_{k+j}=K_{k+j} / N_{1} \ldots N_{k+j}$, we have

$$
\left(1-\frac{\left(\pi c_{k+j} M_{k+j} \lambda\right)^{2}}{3 !}\right)^{2}=\left(1-\frac{\pi^{2}}{6 N_{k+1}^{2} \ldots N_{k+j-1}^{2}} \cdot\left(\frac{K_{k+j} M_{k+j}}{N_{k+j}}\right)^{2} \cdot\left(\frac{\lambda}{N_{1} \ldots N_{k}}\right)^{2}\right)^{2}
$$


We need to ensure all the terms inside the outermost square are positive and their product is strictly positive. For $\lambda \in \mathbf{L}_{k}$, we write

$$
\lambda=\ell_{1}+N_{1} \ell_{2}+\ldots .+\left(N_{1} \ldots N_{k-1}\right) \ell_{k}, \text { for some } \ell_{i} \in L_{i} .
$$

From $N_{i}=M_{i} K_{i}+\alpha_{i}$, we have $\ell_{i} \leq M_{i}-1<N_{i}$ and thus

$$
\begin{aligned}
\frac{\lambda}{N_{1} \ldots N_{k}} & =\frac{\ell_{k}}{N_{k}}+\frac{\ell_{k-1}}{N_{k} N_{k-1}}+\ldots+\frac{\ell_{1}}{N_{k} \ldots N_{1}} \\
& <\frac{M_{k}}{N_{k}}+\frac{M_{k-1}}{N_{k} N_{k-1}}+\ldots+\frac{M_{1}}{N_{k} \ldots N_{1}} \\
& \leq \frac{1}{K_{k}}+\frac{1}{N_{k} K_{k-1}}+\ldots+\frac{1}{N_{k} \ldots N_{2} K_{1}} \\
& \leq \frac{1}{K_{k}}\left(1+\frac{1}{M_{k} K_{k-1}}+\frac{1}{M_{k} N_{k-1} K_{k-2}}+\ldots+\frac{1}{M_{k} N_{k-1} N_{k-2} \ldots N_{2} K_{1}}\right) \\
& \leq \frac{2}{K_{k}} .\left(\text { since all } M_{j}, N_{j} \geq 2\right)
\end{aligned}
$$

For the term $j>1$ in (3.6), we use

$$
\frac{\lambda}{N_{1} \ldots N_{k}}<1, \text { and } \frac{K_{k+j} M_{k+j}}{N_{k+j}} \leq 1
$$

With $N_{j} \geq 2$ for all $j$, we have

$$
(3.6) \geq\left(1-\frac{\pi^{2}}{6 \cdot 2^{2(j-1)}}\right)^{2}, \text { for } j>1 .
$$

If $j=1$,

$$
\left(1-\frac{\pi^{2}}{6} \cdot\left(\frac{K_{k+j} M_{k+j}}{N_{k+j}}\right)^{2} \cdot\left(\frac{\lambda}{N_{1} \ldots N_{k}}\right)^{2}\right)^{2} \geq\left(1-\frac{\pi^{2}}{6} \cdot\left(\frac{2}{K_{k}}\right)^{2}\right)^{2} \geq\left(1-\frac{2 \pi^{2}}{3 K_{k}^{2}}\right)^{2}
$$

Note that our assumption that $\sum_{k=1}^{\infty} \frac{\alpha_{k} \sqrt{M_{k}}}{K_{k}}<\infty$ implies that $K_{k}$ tends to infinity. Hence, there exists $k_{0}$ such that for all $k \geq k_{0}, K_{k} \geq 3$. This ensure the term insider the square is greater than or equal to $\delta:=1-2 \pi^{2} / 27>0$. Putting all the inequality back to (3.5), we obtain

$$
\left|\widehat{\mu_{>k}}(\lambda)\right|^{2} \geq \delta^{2} \cdot \prod_{j=2}^{\infty}\left(1-\frac{\pi^{2}}{6 \cdot 2^{2(j-1)}}\right)^{2}:=c_{0} .
$$

Hence, $\delta(\Lambda) \geq c_{0}$. As $\sum_{j=2}^{\infty} \pi^{2} /\left(6 \cdot 2^{2(j-1)}\right)<\infty$ and $\pi^{2} /\left(6 \cdot 2^{2(j-1)}\right)<1$ for all $j>1, c_{0}>0$ and this completes the proof. 
Proof of Theorem 1.3. (a) follows directly from Theorem 3.3. For (b), Proposition 3.4 implies that $\delta(\Lambda)>0$ and hence the measure $\mu$ is frame spectral by Theorem 3.3.

\section{Non-SPeCtral MeAsures}

In this section, we will see the measures defined by the almost-Parseval-frame tower in Theorem 1.2 is in general not spectral. For a given probability measure $\mu$, we let

$$
Z(\widehat{\mu})=\{\xi \in \mathbb{R}: \widehat{\mu}(\xi)=0\}
$$

be its zero set of $\widehat{\mu}$. We recall that the collection of the exponentials $\left\{e^{2 \pi i \lambda x}: \lambda \in \Lambda\right\}$ is a mutually orthogonal set if the exponential functions are mutually orthogonal in $L^{2}(\mu)$. In order to show $\mu$ cannot be a spectral measure, we need the following simple observation.

Lemma 4.1. If $\mu$ is a spectral measure whose support is an infinite set, then any mutually orthogonal set $\Lambda$ must be of infinite cardinality and satisfies $\Lambda-\Lambda \subset$ $Z(\widehat{\mu}) \cup\{0\}$.

Proof. If $\mu$ is a spectral measure whose support is an infinite set, then $L^{2}(\mu)$ is of infinite dimension as a vector space, so any mutually orthogonal sets must be infinite in cardinality. For mutually orthogonality to hold, we need for any $\lambda \neq \lambda^{\prime} \in \Lambda$,

$$
0=\int e^{2 \pi i\left(\lambda-\lambda^{\prime}\right)} x d \mu(x)=\widehat{\mu}\left(\lambda-\lambda^{\prime}\right)
$$

Hence, $\Lambda-\Lambda \subset Z(\widehat{\mu}) \cup\{0\}$ follows.

Focusing on the tower we constructed in Theorem 1.2,

$$
N_{j}=K_{j} M_{j}+\alpha_{j} .
$$

and $B_{j}=\left\{0, K_{j}, \ldots,\left(M_{j}-1\right) K_{j}\right\}, L=\left\{0,1, \ldots, M_{j}-1\right\}$, we have

Lemma 4.2.

$$
Z(\widehat{\mu})=\bigcup_{j=1}^{\infty} Z\left(\widehat{\nu}_{j}\right)=\bigcup_{j=1}^{\infty}\left[\frac{N_{1} \ldots N_{j}}{K_{j} M_{j}}\left(\mathbb{Z} \backslash M_{j} \mathbb{Z}\right)\right]
$$

Proof. we can compute directly the zero set of the Fourier transform of $\nu_{j}$ as

$$
\begin{aligned}
\widehat{\nu_{j}}(\xi) & =\frac{1}{M_{j}} \sum_{k=0}^{M_{j}-1} e^{-2 \pi i k K_{j} \xi / N_{1} \ldots N_{j}} \\
& = \begin{cases}\frac{1}{M_{j}} e^{\pi i c_{j}\left(M_{j}-1\right) \xi \frac{\sin \pi c_{j} M_{j} \xi}{\sin \pi c_{j} \xi},} & \text { if } \xi \notin \frac{1}{c_{j}} \mathbb{Z} ; \\
1, & \text { if } \xi \in \frac{1}{c_{j}} \mathbb{Z} .\end{cases}
\end{aligned}
$$


where $c_{j}=K_{j} / N_{1} \ldots N_{j}$. It follows directly that

$$
Z\left(\widehat{\nu}_{j}\right)=\frac{1}{c_{j} M_{j}}\left(\mathbb{Z} \backslash M_{j} \mathbb{Z}\right)=\frac{N_{1} \cdots N_{j}}{K_{j} M_{j}}\left(\mathbb{Z} \backslash M_{j} \mathbb{Z}\right)
$$

so that

$$
Z(\widehat{\mu})=\bigcup_{j=1}^{\infty} Z\left(\widehat{\nu}_{j}\right)=\bigcup_{j=1}^{\infty}\left[\frac{N_{1} \ldots N_{j}}{K_{j} M_{j}}\left(\mathbb{Z} \backslash M_{j} \mathbb{Z}\right)\right]
$$

It is natural to conjecture that

Conjecture 4.3. Suppose that $\left(N_{j}, B_{j}\right)$ are the almost-Parseval-frame tower defined in Theorem 1.2 and the associated measure $\mu$ is spectral. Then all $\alpha_{j}=0$.

However, this will let us into rather involved number theoretic and combinatoric questions. To serve the purpose of this paper, the following proposition shows that under simple conditions on $M_{j}, K_{j}$ and $\alpha_{j}$, the measure $\mu$ cannot be spectral.

Proposition 4.4. Suppose that $\Lambda$ is a mutually orthogonal set for $\mu$ defined in (1.3) with $N_{j}=K_{j} M_{j}+1\left(\alpha_{j}=1\right)$ and $B_{j}=\left\{0, K_{j}, \ldots,\left(M_{j}-1\right) K_{j}\right\}, L_{j}=\left\{0,1, \ldots, M_{j}-1\right\}$ satisfying

(1) all $M=2$ and

(2) all $K_{j}$ are odd;

Then

$$
\# \Lambda \leq 2
$$

Proof. Suppose that there exists mutually orthogonal set $\Lambda$ with cardinality greater than 2. We can find distinct $\lambda_{1}, \lambda_{2}, \lambda_{3}$ such that $\lambda_{1}-\lambda_{2}, \lambda_{3}-\lambda_{2}, \lambda_{1}-\lambda_{3} \in Z(\widehat{\mu})$. Hence, we can write

$$
\begin{gathered}
\lambda_{1}-\lambda_{2}=\frac{\left(N_{1} \ldots N_{j}\right)}{K_{j} M_{j}}\left(r_{j}+M_{j} q_{j}\right), \lambda_{3}-\lambda_{2}=\frac{\left(N_{1} \ldots N_{k}\right)}{K_{k} M_{k}}\left(r_{k}+M_{k} q_{k}\right), \\
\lambda_{1}-\lambda_{3}=\frac{\left(N_{1} \ldots N_{\ell}\right)}{K_{\ell} M_{\ell}}\left(r_{\ell}+M_{\ell} q_{\ell}\right)
\end{gathered}
$$

where $0<r_{n}<M_{n}$ for $n=j, k, \ell$ and $q_{j}, q_{k}, q_{\ell}$ are integers. Denote by

$$
\mathbf{N}_{n}=N_{1} \ldots N_{n} \text {. }
$$

As $\left(\lambda_{1}-\lambda_{2}\right)-\left(\lambda_{3}-\lambda_{2}\right)=\lambda_{1}-\lambda_{3}$, we have the following algebraic relation,

$$
\frac{\mathbf{N}_{j}}{K_{j} M_{j}}\left(r_{j}+M_{j} q_{j}\right)-\frac{\mathbf{N}_{k}}{K_{k} M_{k}}\left(r_{k}+M_{k} q_{k}\right)=\frac{\mathbf{N}_{\ell}}{K_{\ell} M_{\ell}}\left(r_{\ell}+M_{\ell} q_{\ell}\right) .
$$

It follows that

$$
\mathbf{N}_{j} K_{k} K_{\ell} M_{k} M_{\ell}\left(r_{j}+M_{j} q_{j}\right)-\mathbf{N}_{k} K_{j} K_{\ell} M_{j} M_{\ell}\left(r_{k}+M_{k} q_{k}\right)=\mathbf{N}_{\ell} K_{j} K_{k} M_{j} M_{k}\left(r_{\ell}+M_{\ell} q_{\ell}\right) .
$$


Hence,

$$
\begin{aligned}
& \mathbf{N}_{j} K_{k} K_{\ell} M_{k} M_{\ell} r_{j}-\mathbf{N}_{k} K_{j} K_{\ell} M_{j} M_{\ell} r_{k}-\mathbf{N}_{\ell} K_{j} K_{k} M_{j} M_{k} r_{\ell} \\
= & M_{j} M_{k} M_{\ell} \cdot\left(\mathbf{N}_{\ell} K_{j} K_{k} q_{\ell}-\mathbf{N}_{k} K_{j} K_{\ell} q_{k}-\mathbf{N}_{j} K_{k} K_{\ell} q_{j}\right) .
\end{aligned}
$$

In the first case, if all $M_{j}=2$, then all $0<r_{i}<2$ which means all $r_{i}=1$. (4.1) is reduced to

$$
\mathbf{N}_{j} K_{k} K_{\ell}-\mathbf{N}_{k} K_{j} K_{\ell}-\mathbf{N}_{\ell} K_{j} K_{k}=2 \cdot\left(\mathbf{N}_{\ell} K_{j} K_{k} q_{\ell}-\mathbf{N}_{k} K_{j} K_{\ell} q_{k}-\mathbf{N}_{j} K_{k} K_{\ell} q_{j}\right) .
$$

The right hand side is an even number. However, as all $K_{j}$ are odd numbers, and all $N_{j}=2 K_{j}+1$ are odd, each term in the left hand side of (4.1) must be odd and hence the left hand side is an odd number overall. This is a contradiction. Hence, we cannot have a mutually orthogonal set of cardinality large than 2 .

We are now ready to construct the example in Theorem 1.4.

Proof of Theorem 1.4. Let $p$ be a prime number of the form $4 k+3$. It is well known that there are infinitely many primes of such form by the Dirichlet theorem. Writing

$$
p^{j}=2 K_{j}+1
$$

j we claim that $K_{j}$ is an odd number whenever $j$ is odd. Expanding in binomial theorem, we have for some integer $L_{j}$,

$$
K_{j}=\frac{p^{j}-1}{2}=\frac{(4 k+3)^{j}-1}{2}=\frac{4 L_{j}+3^{j}-1}{2} .
$$

It suffices to show that $\left(3^{j}-1\right) / 2$ is an odd number if $j$ is odd. But from Binomial expansion, $\frac{3^{j}-1}{2}=\frac{(2+1)^{j}-1}{2}=2^{j-1}+j 2^{j-2}+\ldots\left(\begin{array}{l}j \\ 2\end{array}\right) 2+j$. This shows that $\frac{3^{j}-1}{2}$ is an odd number.

Letting $N_{j}=p^{2 j-1}=2 K_{2 j-1}+1$ where $p$ is a prime number of the form $4 k+3$ and $B_{j}=\left\{0, K_{2 j-1}\right\}$. From Example 2.3, we have an almost-Parseval-frame tower. By Theorem 1.3(b), the associated measure $\mu$ is frame spectral. On the other hand, it is non-spectral by Proposition 4.4.

We end this section with a remark.

Remark 4.5. In [HLL], it was proved that if $\nu$ is a spectral measure on $[0,1]$ with spectrum inside $\mathbb{Z}$, then any $\mu=\nu * \delta_{A}$, with $A \subset \mathbb{Z}$, is a frame spectral measure and some of them are not spectral. In view of Theorem 1.2, the measure $\mu$ we constructed cannot be of the form $\nu * \delta_{A}$, where $\nu$ is spectral and $\delta_{A}$ is a discrete measure supported on some set $A$. If it was the case, then

$$
\widehat{\mu}(\xi)=\widehat{\nu}(\xi) \widehat{\delta_{A}}(\xi)
$$


and this would have implied that any mutually orthogonal set of $\nu$ must be mutually orthogonal set of $\mu$ and hence cardinality of such sets for $\mu$ for be infinite, which is a contradiction by Proposition 4.4 we just proved.

\section{Appendix: HausdorfF Dimension}

In this section, we study the Hausdorff dimension, denoted by $\operatorname{dim}_{H}$, of the support of $\mu$, which is an important question in fractal geometry. We refer the reader to [Fal] the definition of Hausdorff dimension. Given a sequence of positive integers $M_{j}$ and a sequence of numbers $r_{j}$. Suppose that $0<r_{j}<1, M_{j} \geq 2$ and $r_{j} M_{j} \leq 1$ for all $j$. For $k \geq 1$, we let $D_{0}=\emptyset, D_{k}=\left\{\left(i_{1}, \ldots, i_{k}\right): i_{j} \in\left\{0,1, \ldots, M_{j}-1\right\}\right\}$. For $\mathbf{i} \in D_{k}$ and $\mathbf{j} \in D_{\ell}, \mathbf{i j} \in D_{k+\ell}$ is the standard concatenation of two words. For each $\sigma \in \bigcup_{k=1}^{\infty} D_{k}$, we define an interval $J_{\sigma}$. We say that

$$
E=\bigcap_{k=1}^{\infty} \bigcup_{\sigma \in D_{k}} J_{\sigma}
$$

is a homogeneous Moran set if the following conditions are satisfied.

(1) $J_{\emptyset}=[0,1]$. For any $\sigma \in D_{k}, J_{\sigma 0}, \ldots, J_{\sigma\left(M_{k+1}-1\right)}$ are subinterval of $J_{\sigma}$ enumerated from left to right and $J_{\sigma i} \cap J_{\sigma j}$ has intersects at most one point.

(2) For any $k \geq 1$, for any $\sigma \in D_{k-1}$ and $j \in\left\{0,1, \ldots, M_{k}-1\right\}$,

$$
r_{k}=\frac{\left|J_{\sigma j}\right|}{\left|J_{\sigma}\right|} \text {. }
$$

$|\cdot|$ denotes the Lebesgue measure of the interval.

(3) For any $\sigma \in D_{k}$, the gaps between $J_{\sigma i}$ and $J_{\sigma(i+1)}$ are equal in length, the left endpoint of $J_{\sigma 0}$ is equal to the left endpoint of $J_{\sigma}$ and the right endpoint of $J_{\sigma\left(M_{k+1}-1\right)}$ is equal to the right endpoint of $J_{\sigma}$.

It was shown that [FWW] (see also [FLW, Proposition 3.1]) that the Hausdorff dimension of $E$ is equal to

$$
\operatorname{dim}_{H}(E)=\liminf _{j \rightarrow \infty} \frac{\log \left(M_{1} \ldots M_{j}\right)}{-\log \left(r_{1} \ldots r_{j}\right)} .
$$

Turning to our case where $N_{j}=M_{j} K_{j}+\alpha_{j}$ and $B_{j}=\left\{0, K_{j}, \ldots, K_{j}\left(M_{j}-1\right)\right\}$, the support of the measure $\mu$ is

$$
K_{\mu}=\left\{\sum_{j=1}^{\infty} \frac{i_{j} K_{j}}{N_{1} \ldots N_{j}}: i_{j} K_{j} \in B_{j}\right\}=\bigcap_{k=1}^{\infty} \bigcup_{\sigma \in D_{k}} J_{\sigma}
$$

where $J_{\sigma}=\left[\sum_{j=1}^{k} i_{j} K_{j}\left(N_{1} \ldots N_{j}\right)^{-1}, \sum_{j=1}^{k} i_{j} K_{j}\left(N_{1} \ldots N_{j}\right)^{-1}+\left(N_{1} \ldots N_{k}\right)^{-1}\right]$ and $\sigma=$ $\left(i_{1}, \ldots, i_{k}\right)$. Note that The support $K_{\mu}$ is contained in the interval $[0, \rho]$, where $\rho=\sum_{j=1}^{\infty}\left(K_{j}\left(M_{j}-1\right)\right)\left(N_{1} \ldots N_{j}\right)^{-1}$. By a simple rescaling, $C=\rho^{-1} K_{\mu}$. It is easy 
to see that $C$ is actually a homogeneous Moran set with $r_{1}=1 / N_{1}$ and $r_{k}=$ $\left(N_{1} \ldots N_{k}\right)^{-1} /\left(N_{1} \ldots N_{k-1}\right)^{-1}=1 / N_{k}$. Hence, we have thus proved

\section{Proposition 5.1.}

$$
\operatorname{dim}_{H}\left(K_{\mu}\right)=\liminf _{j \rightarrow \infty} \frac{\log \left(M_{1} \ldots M_{j}\right)}{\log \left(N_{1} \ldots N_{j}\right)}
$$

We now compute the Hausdorff dimension of some frame spectral measures $\mu$.

Example 5.2. In Example 2.3, $N_{j}=p^{j}=2 K_{j}+1$ and $M_{j}=2$ for all $j$. In this case, the Hausdorff dimension

$$
\operatorname{dim}_{H}\left(K_{\mu}\right)=\liminf _{j \rightarrow \infty} \frac{\log 2^{j}}{\log \left(p^{1+2+\ldots+j}\right)}=\liminf _{j \rightarrow \infty} \frac{2 j \log 2}{j(j+1) \log p}=0 .
$$

The non-spectral measure with Fourier frame given in Theorem 1.4 is a special case of this type, and thus the support has Hausdorff dimension 0.

This example shows that frame spectral measure can be very " thin", similar situation happens for spectral measures [DaS]. The following example shows that our construction does give frame spectral measures with positive Hausdorff dimension.

Example 5.3. In Example 2.4, $M_{j}=K_{j}^{\beta}$ and $\alpha_{j}=1$ (i.e. $\gamma=0$ ) for all $j$. Then $N_{j}=K_{j}^{1+\beta}+1$. In this case, the Hausdorff dimension

$$
\operatorname{dim}_{H}\left(K_{\mu}\right)=\liminf _{j \rightarrow \infty} \frac{\log \left(\left(K_{1} \ldots K_{j}\right)^{\beta}\right)}{\log \left(\left(K_{1}^{1+\beta}+1\right) \ldots\left(K_{j}^{1+\beta}+1\right)\right)} .
$$

As $\lim _{x \rightarrow \infty} \log (1+x) / \log x=1$, for $x$ large, $C^{-1} \log x \leq \log (1+x) \leq C \log x$ for some constant $C>0$. Hence, if $K_{j}$ is large enough,

$$
C^{-1} \log \left(\left(K_{1} \ldots K_{j}\right)^{1+\beta}\right) \leq \log \left(\left(K_{1}^{1+\beta}+1\right) \ldots\left(K_{j}^{1+\beta}+1\right)\right) \leq C \log \left(\left(K_{1} \ldots K_{j}\right)^{1+\beta}\right)
$$

This implies that

$$
\begin{aligned}
\frac{\beta}{C(1+\beta)}=\liminf _{j \rightarrow \infty} \frac{\beta \log \left(\left(K_{1} \ldots K_{j}\right)\right)}{C(1+\beta) \log \left(\left(K_{1} \ldots K_{j}\right)\right)} & \leq \operatorname{dim}_{H}\left(K_{\mu}\right) \\
& \leq \liminf _{j \rightarrow \infty} \frac{\beta \log \left(\left(K_{1} \ldots K_{j}\right)\right)}{C^{-1}(1+\beta) \log \left(\left(K_{1} \ldots K_{j}\right)\right)} \\
& =\frac{\beta}{(1+\beta) C^{-1}} .
\end{aligned}
$$

Hence, the support of the frame spectral measure has Hausdorff dimension at least $\frac{\beta}{C(1+\beta)}>0$. 


\section{REFERENCES}

[AH] L.-X An And X.-G He, A class of spectral Moran measures. J. Funct. Anal. 266 (2014), 343-354.

[Chr] O. Christensen, An Introduction to Frames and Riesz Bases, Appl. Numer. Harmon. Anal.. Birkhäuser Boston Inc., Boston, MA, 2003.

[CK] P. Casazza, G. Kutyniok (Eds.), Finite Frames: Theory and Applications, Appl. Numer. Harmon. Anal., Birkhäuser/Springer, New York, 2013.

[DaHL] X.-R. DAI, X.-G He And C.-K LAI, Spectral property of Cantor measures with consecutive digits, Adv. Math., 242 (2013), 187-208.

[DaS] X.-R. DAI, Q.-Y Sun, Spectral measures with arbitrary Hausdorff dimensions, J.Funct.Anal., 268 (2015),2464-2477.

[DHS] D. Dutkay, D. Han And Q. Sun, Divergence of mock and scrambled Fourier series on fractal measures, Trans. Amer. Math. Soc., 366(2014), 2191-2208.

[DHSW] D. Dutkay, D.-G. Han, Q. Sun and E. Weber, On the Beurling dimension of exponential frames, Adv in Math., 226 (2011), 285-297.

[DHW1] D. Dutkay, D.-G. Han, And E. WeBer, Bessel sequence of exponential on fractal measures, J. Funct. Anal., 261 (2011), 2529-2539.

[DHW2] D. Dutkay, D. Han, And E. Weber, Continuous and discrete Fourier frames for Fractal measures, Tran Amer Math Soc., 366 (2014), 1213-1235.

[DJ1] D. Dutkay and P. Jorgensen, Fourier frequencies in affine iterated function systems, J. Funct. Anal., 247 (2007), 110 - 137.

[DL1] D. Dutkay And C.-K. LaI, Uniformity of measures with Fourier frames, Adv. Math., 252 (2014), 684-707.

[DL2] D. DutKay And C.-K. LAI, Self-affine spectral measures and frame spectral measures on $\mathbb{R}^{d}$, submitted.

[DS] R. Duffin, And A. Schaeffer, A class of nonharmonic Fourier series, Tran. Amer. Math. Soc., 72 (1952), 341-366.

[Fal] K. FAlCOner,, Fractal geometry. Mathematical foundations and applications, Second edition. John Wiley and Sons, Inc., Hoboken, NJ, 2003.

[Fu] B. Fuglede, Commuting self-adjoint partial differential operators and a group theoretic problem, J. Funct. Anal., 16 (1974), 101-121.

[FL] D.-J FENG AND K.-S LAU, Multifractal formalism for self-similar measures with weak separation condition, J. Math. Pures Appl. 92 (2009), 407-428.

[FLW] D.-J Feng, K.-S Lau And J. Wu, Ergodic limits on the conformal repellers, Adv. Math., 169 (2002), 58-91.

[FWW] D.-J. Feng, Z.-Y. Wen, J. Wu, Some dimensional results for homogeneous Moran sets, Sci. China Ser. A, 40 (1997), 475-482.

[GL] J.-P Gabardo AND C.-K. LAI, Spectral measures associated with the factorization of the Lebesgue measure on a set via convolution, J. Fourier. Anal. Appl., 20 (2014), 453-475.

[HLL] X.-G. He, C.-K. Lai And K.-S. LaU, Exponential spectra in $L^{2}(\mu)$, Appl. Comput. Harmon. Anal., 34 (2013), 327-338.

[IKT1] A. Iosevich, N. Katz And T. TaO, Convex bodies with a point of curvature do not have Fourier bases, Amer. J. Math., 123 (2001), 115-120.

[IKT2] A. Iosevich, N. Katz, T. Tao, Fuglede Spectral Conjecture holds for convex planar domain, Math. Res. Letter, 10(2003), 559-569.

[JP1] P. Jorgensen and S. Pedersen, Dense analytic subspaces in fractal $L^{2}$ spaces., J. Anal. Math., 75 (1998), 185-228. 
[K] M. Kolountzakis, Multiple lattice tiles and Riesz bases of exponentials, Proc. Amer. Math. Soc. 143 (2015), 741-747.

[KN] Gady Kozma, Shahaf Nitzan, Combining Riesz bases, Invent. math., 199 (2015), 267285.

[Lai] C.-K. LAI, On Fourier frame of absolutely continuous measures, J. Funct. Anal., 261 (2011), 2877-2889.

[ŁaW1] I. ŁabA And Y. Wang, On spectral Cantor measures, J. Funct. Anal., 193 (2002), 409 420.

[ŁP1] I. Łaba And M. Pramanik, Arithmetic Progressions in sets of fractional dimensions, Geo. Funct. Anal., 19 (2009), 429-456.

[ŁP2] I. ŁabA And M. Pramanik, Maximal operators and differentiation theorems for sparse sets, Duke Math. J., 158 (2011), 347 - 411.

[MSS] A. Marcus, D. Spielman and N. Srivastava, Interlacing families II: Mixed characteristic polynomials and the Kadison-Singer Problem, Ann of Math., 182 (2015), 327-350.

[NOU] S. Nitzan, A. OlevskiI, A. Ulanovskis, Exponential frames on unbounded sets, preprint.

[St1] R. StRIChartz, Mock Fourier series and transforms associated with certain Cantor measures, J. Anal. Math., 81 (2000), 209-238.

[St2] R. Strichartz, Convergence of Mock Fourier series, J. Anal. Math., 99 (2006), 333-353.

[T] T. TAO, Fuglede's conjecture is false in 5 or higher dimensions, Math. Res. Letter, 11 (2004), 251-258.

[W] Y. WANG, Wavelets, tiling, and spectral sets. Duke Math. J. 114 (2002), no. 1, 43-57.

Department of Mathematics, San Francisco State University, 1600 Holloway AvEnUe, San Francisco, CA 94132.

E-mail address: cklai@sfsu.edu

Department of Mathematics, Hong Kong University of Science and Technology, HONG KONG

E-mail address: yangwang@ust.hk 